\title{
Mortalidad y carga de muertes prematuras por flebitis y tromboflebitis, Cuba años 2000 y 2005
}

\section{Mortality and burden of premature deaths from phlebitis and thrombophlebitis in Cuba in the years 2000 and 2005}

\author{
Ulises de J esús Gallardo Pérez'; Armando H. Seuc J o"; Leonardo Zangronis \\ Ruano'"'; Nelson Chirino Carreño' \\ 'Especialista de I Grado en Medicina General Integral. Investigador Auxiliar. \\ Instituto Nacional de Angiología y Cirugía Vascular. La Habana, Cuba. \\ "Doctor en Ciencias. Investigador Titular. Instituto Nacional de Angiología y Cirugía \\ Vascular. La Habana, Cuba. \\ "'Especialista de I Grado en Medicina General Integral. Policlínico "Hermanos Ruiz \\ Aboy". La Habana, Cuba. \\ IVEspecialista de II Grado en Angiología y CirugíaVascular. Profesor Auxiliar. \\ Instituto Nacional de Angiología y Cirugía Vascular. La Habana, Cuba.
}

\section{RESUMEN}

Introducción La flebitis y tromboflebitis de los miembros inferiores es una de las causas básicas de muerte por enfermedades vasculares periféricas y uno de los principales motivos de ingreso en los servicios de angiología del país.

Objetivos Identificar la mortalidad y la carga por muertes prematuras a consecuencia de la flebitis y tromboflebitis en la población cubana.

Métodos Se realizó un estudio descriptivo retrospectivo de la mortalidad por flebitis y tromboflebitis en miembros inferiores en los años 2000 y 2005 según la base de datos de la Dirección Nacional de Estadística. Se realizó el cálculo de las tasas brutas y específicas de mortalidad por edad, sexo y la tasa de años de vida potenciales perdidos por muertes prematuras según sexo y provincias.

Resultados La mayor tasa de mortalidad y de años de vida potenciales perdidos fue en el año 2005, en el grupo de edad de 60 años y más y en el sexo femenino. Incrementaron la carga por muertes prematuras las provincias de Ciego de Ávila, 
Camagüey, Villa Clara, Cienfuegos, Santiago de Cuba, Pinar del Río, Matanzas, Isla de la Juventud y Ciudad de La Habana.

Conclusiones Estos resultados ofrecen una visión más amplia del impacto de la trombosis venosa en los miembros inferiores sobre el patrón de mortalidad del país. La carga por muertes prematuras constituye un elemento más, para planificar e implementar las políticas de salud relativas a las enfermedades venosas de los miembros inferiores.

Palabras clave: Mortalidad, carga por muertes prematuras, flebitis y tromboflebitis, Epidemiología, Cuba.

\section{ABSTRACTS}

Introduction Phlebitis and thrombophlebitis of the lower extremities is one of the basic causes of death from peripheral vascular diseases and one of the main reasons for admission to the Angiology Services throughout the country.

Objectives To identify mortality rates and burden of premature deaths from phlebitis and thrombophlebitis occurred in the Cuban population.

Méthods A retrospective descriptive study of mortality from phlebitis and thrombophlebitis of lower extremities in Cuba in the years 2000 and 2005 was conducted, taking data from the National Division of Statistics as a basis. Gross and specific mortality rates by sex and age, and the rate of potential lost years of life caused by premature deaths by sex and province were estimated.

Results The highest mortality rate and potential lost years of life rate were found in the over 60 years-old group and the female sex in the year 2005. The burden of premature deaths increased in Ciego de Avila, Camaguey, Villa Clara, Cienfuegos, Santiago de Cuba, Pínar del Río, Matanzas, Isla de la Juventud and Ciudad de la Habana provinces.

Conclusions These results provide a broader overview of the impact of venous thrombosis on the lower limbs according to the national mortality patterns. The burden of premature deaths is another element for the planning and implementation of health policies related to venous diseases in the lower extremities.

Key words: Mortality, burden of premature deaths, phlebitis and thrombophlebitis, Epidemiology, Cuba.

\section{NTRODUCCI ÓN}

La flebitis es la inflamación de una vena y la tromboflebitis está relacionada con un coágulo de sangre en la vena y entre los factores de riesgo se registra el encamamiento prolongado, trastornos de la coagulación de la sangre, antecedentes de cirugía, neoplasias malignas y otros. ${ }^{1}$

Esta entidad es uno de los motivos de ingreso en los servicios de angiología del país y del mundo por las consecuencias fatales que pueden originarse a partir de su 
aparición. Requiere de un tratamiento oportuno, por constituir una de las causas básicas de muertes por enfermedades del sistema circulatorio. ${ }^{2}$ Dentro de esta categoría quedan incluidas las flebitis y tromboflebitis superficiales y profundas de miembros inferiores, estas últimas son las más peligrosas, pues dan lugar a los tromboembolismos pulmonares, lo que conlleva a complicaciones fatales. ${ }^{3}$

Un estudio realizado por el Instituto Nacional de Angiología y Cirugía Vascular sobre la carga por muertes prematuras para un grupo de enfermedades vasculares periféricas en el año 2000, encontró que la flebitis y tromboflebitis ocuparon el 8 vo. lugar como causa básica de muerte en la población cubana dentro del grupo de afecciones circulatorias periféricas.

Si bien es cierto que la flebitis y tromboflebitis de los miembros no aparecen dentro de las primeras causas de muerte en los estudios de mortalidad realizados en el país, tampoco existen estudios epidemiológicos sobre su impacto en la población cubana, a pesar de ser un motivo de ingreso frecuente en los servicios de angiología. ${ }^{4}$

En el ámbito internacional los estudios de mortalidad por enfermedades circulatorias no son tan específicos sobre entidades vasculares periféricas, hay referencias con respecto a la aterosclerosis, aneurismas, angiopatía periférica diabética y otras causas de muerte.

La mayoría de los estudios de trombosis venosa aparecidos en la literatura se limitan fundamentalmente a su morbilidad a nivel de instituciones de salud, por tanto es importante adentrarse en el tema para aportar algunos elementos sobre la epidemiología de esta entidad.

La mortalidad y los años de vida potenciales perdidos (AVPP), son indicadores que permiten estudiar el estado de salud de la población. Son indicadores de salud negativo, pero no se puede prescindir de su utilización en el proceso de planificación de los recursos, programas de salud y para la toma de decisiones. ${ }^{5}$

Precisamente este análisis de la mortalidad se hace a partir de las causas básicas de muerte ya que desde el punto de vista de la prevención de la mortalidad temprana, es importante cortar la cadena de acontecimientos o instituir la curación y prevenir que opere la causa precipitante.

En la Clasificación Estadística Internacional de las Enfermedades y Problemas Relacionados con la Salud ( $\mathrm{CIE}$ ), se registra a la flebitis y tromboflebitis como una causa básica de muerte. Por tanto en la medida que se pueda aplicar acciones de salud más efectivas, debe disminuir el riesgo de morir a consecuencia de este evento de salud. ${ }^{4}$

Los objetivos de este trabajo son cuantificar la mortalidad y la carga por muertes prematuras a consecuencia de la flebitis y tromboflebitis de miembros inferiores en Cuba en los años 2000 y 2005.

\section{MÉTODOS}

Se realizó un estudio descriptivo retrospectivo sobre la mortalidad por flebitis y tromboflebitis de miembros inferiores en Cuba en los años 2000 y 2005 . Se estudiaron las defunciones registradas por la Dirección Nacional de Estadística 
sobre la base de la 9na y 10ma Revisión de la CIE para los años 2000, y respectivamente identificados con los códigos 451 y 180 . Esta categoría incluye las flebitis superficiales y profundas de miembros inferiores.

Para el análisis estadístico se utilizaron la frecuencia absoluta, tasa bruta de mortalidad y la tasa de AVPP para Cuba, según provincias y sexo, así como las tasas específicas de mortalidad por edad y sexo. Para el cálculo de la carga por muertes prematuras se utilizó el indicador de los AVPP y se realizó multiplicando el número de defunciones para cada grupo de edad y sexo por el factor de conversión correspondiente. El factor de conversión son los años perdidos para cada grupo de edad y sexo. Se utilizó la esperanza de vida de Cuba para el año 2000 y las proyecciones de población del año 2000 y 2005 ofrecidas por la Oficina Nacional de Estadística.

\section{RESULTADOS}

Se encontró que la mortalidad por flebitis y tromboflebitis en la población cubana es baja para ambos años, pero muy discretamente superior en el año 2005 con respecto al año 2000. Es decir, en el 2000 hubo 44 defunciones registradas por flebitis y tromboflebitis para una tasa de $0,3 \times 100000$ habitantes, mientras en el 2005 fueron 73 fallecidos para una tasa de 0,6 por cada 100000 habitantes.

En cuanto a los grupos de edades (figura 1), se observa para ambos años que existe un mayor riesgo de morir en las personas de edad avanzada y a su vez se constata una tendencia al incremento de la mortalidad en la medida que avanza la edad del individuo.

Respecto al sexo se registró en el año 2000 una tasa de mortalidad similar para ambos sexos (0,3 x 100000 habitantes), no así en el año 2005 donde la tasa de mortalidad fue superior para el sexo femenino $(0,5$ por 100000 hombres y 07 por 100000 mujeres).

En cuanto a los AVPP, se pudo apreciar un mayor número de AVPP en el año 2005, es decir, del 2000 al 2005 hubo un incremento de la mortalidad prematura por esta afección circulatoria (5,4 y 10,6 por 100000 habitantes, repectivamente). En el sexo femenino hubo una mayor tasa de AVPP para ambos años.

Con respecto a la tasa de mortalidad por flebitis y tromboflebitis por provincias (figura 2), en el año 2000 las provincias con mayor carga fueron Ciudad de La Habana, Granma y Sancti Spíritus, sin embargo no se registraron fallecidos en Matanzas, Cienfuegos, Ciego de Avila e Isla de la Juventud. Por otra parte, en el año 2005 hubo nueve provincias que incrementaron la carga por muertes prematuras con respecto al año 2000: Ciego de Avila, Camagüey, Villa Clara, Cienfuegos, Santiago de Cuba, Pinar del Río, Matanzas, I sla de la Juventud y Ciudad de La Habana; las mayores tasas de mortalidad prematura correspondieron a las provincias de Ciego de Ávila, Camagüey, I sla de la Juventud y Ciudad de La Habana, mientras en Las Tunas no se reportaron fallecidos por esta causa en el 2005. Es decir, no hubo semejanzas en los territorios más afectados entre ambos años objeto de estudio.

También sería válido señalar que en las provincias de Ciudad de La Habana, Sancti Spíritus, Holguín, Granma y Guantánamo disminuyó el impacto por muertes prematuras de la flebitis y tromboflebitis en la población. 


\section{DISCUSIÓN}

La flebitis y la tromboflebitis es una enfermedad venosa que tiene como causa el éstasis sanguíneo, el daño vascular o el aumento de la coagulación. Su complicación fundamental es el embolismo pulmonar y el éstasis crónico en las piernas. Está demostrado que la trombosis venosa de miembros inferiores pueden ser un antecedente en los pacientes que se presenta un tromboembolismo pulmonar. ${ }^{6}$

La trombosis de miembros es una afección que se presenta con mayor frecuencia en los individuos de edad avanzada y la literatura científica así lo avala. ${ }^{7}$ En este estudio se mostró que el mayor número de defunciones está concentrado justamente en las personas de mayor edad, donde se describe la mayor incidencia de la tromboflebitis, de ahí la mayor carga por muertes prematuras en las edades más tardías de la vida.

La población senil de Cuba y el mundo tiene una tendencia al incremento en los últimos años, por tanto se augura un incremento de esta afección dentro de la población. ${ }^{4}$ De ahí la necesidad de aplicar con mayor regularidad y efectividad las estrategias de salud individual y colectiva para reducir la incidencia de las flebitis y tromboflebitis y sus posibles complicaciones que puedan llevar a la muerte. ${ }^{8}$ Es pertinente señalar que los adultos mayores suelen estar sometidos a múltiples factores de riesgo como la inmovilización, cirugía, uso de catéteres, quimioterapia, la presencia de neoplasias malignas y otros, que lo hacen más propensos a presentar este tipo de afección vascular. ${ }^{1,9-11}$

Existen normas y conductas demostradas científicamente que contribuyen a la profilaxis de la enfermedad tromboembólica y en la medida que sean ejecutadas precozmente se puede minimizar su impacto por mortalidad dentro de la población. ${ }^{9,10,12,13}$ El sistema venoso de los miembros inferiores es el más proclive a desarrollar enfermedad tromboembólica, por su tendencia a producir insuficiencia venosa, estasis sanguíneo y várices. ${ }^{1}$

Esto requiere de un conocimiento de la historia natural de la enfermedad por parte del personal de la salud encargado de poner en práctica los procederes que impidan el origen de una trombosis de miembros inferiores y a partir de ahí prevenir un desenlace fatal para el paciente. ${ }^{11}$

En cuanto al sexo, está bien descrito que la morbilidad por flebitis y tromboflebitis es más frecuente en el sexo femenino y su mortalidad suele tener un comportamiento similar, lo que se confirmó en los resultados obtenidos para el año 2005, no así en el año 2000, donde el riesgo de morir fue idéntico para ambos sexos. Al respecto se debe señalar que la literatura científica indica una sobremortalidad femenina, por tanto este estudio concuerda en cierta medida con evidencias anteriores nacionales e internacionales. ${ }^{10}$

Los resultados de la carga por muertes prematuras según provincias constituye un aporte al comportamiento epidemiológico de esta afección dentro del país, si bien existen disímiles estudios sobre el comportamiento de las flebitis y tromboflebitis, no se encontraron evidencias sobre estos aspectos de la mortalidad en Cuba y en especial su descripción por provincias, lo cual es muy importante desde la perspectiva de la salud pública que concibe que los problemas de salud no tienen similar comportamiento en todos las regiones y/o provincias del país, pues existen condiciones específicas en cada territorio que determinan el perfil de mortalidad 
independientemente de la división política administrativa establecida. Esto permitiría reflexionar sobre los determinantes de la salud en cada territorio del país e identificar posibles brechas de reducción para lograr estrategias de salud más efectivas y reducir la carga de la flebitis y tromboflebitis en la población cubana.

Finalmente se puede concluir que se consiguió explicar algunas características de la mortalidad por flebitis y tromboflebitis en miembros inferiores y su carga por muertes prematuras en la población cubana. Estos resultados ofrecen una visión más amplia del impacto de la trombosis venosa sobre el patrón de mortalidad de la población y constituyen una certeza útil para el análisis de los decisores y planificadores de la salud con respecto a la enfermedad tromboembólica.

\section{REFERENCI AS BI BLI OGRÁFICAS}

1. Aronés-Valdívia A, Carrasco A, Delgado C, López M. Incidencia de la enfermedad tromboembólica en neoplasias malignas en cuatro hospitales de Lima. Rev Soc Peruana Med Inter. 2002; 15(2).

2- Miranda G, Díaz J, Arencibia P, Díaz C, Vidal A. Tromboembolismo pulmonar: venografía por tomografía computada versus ecografía doppler, para detección de trombosis venosa profunda de extremidades inferiores. Rev Chil Radiol. $2005 ; 11(1): 5-8$.

3- OMS/OPS. Clasificación Estadística Internacional de Enfermedades. Problemas relacionados con la salud. Publicación científica No.554. 3ra ed. Washington,D.C.: OPS; 1997:29-33.

4- Gallardo U, Seuc A, Zangronis L, Puentes I, Rubio Y, Lopez L. Impacto de la mortalidad por efermedades vsculares priféricas Cuba 2000. Rev Cubana Angiol. 2005; 6(1).

5- Astraín M. Indicador Mortalidad. En: Análisis de Situación de Salud. La Habana: Editorial Ciencias Médicas ;2004.p.26-46.

6 - Geerts WH, Pineo GF, Heit JA, Bergqvist D, Lassen MR, Colwell CW, et al. Prevention of venous thromboembolism: the Seventh ACCP Conference on Antithrombotic and Thrombolytic Therapy. Chest. 2004; 126(3 Suppl): 338-400.

7- Murphy PT. Factor V. Leiden and venous thromboembolism. Ann Intern Med. 2004; 141(6): 483-4.

8- Estevan Solano J. Protocolo de patología vascular para atención primaria. Oviedo: Insalud; 2000: 13-25.

9- Mele E. Manejo terapéutico actual del tromboembolismo pulmonar: de la profilaxis a los fibrinolíticos. Rev Soc Parag Cardiol. 2004;2(2): 133-42.

10- Hamilton F, Rollo A. Trombose Venosa Profunda dos membros Inferiores: Incidencia, Patología, Patogenia, Fisiopatología e Diagnóstico. En: Doencas Vasculares Perifèricas. 3era ed. Medsi. 2002: 970.

11-Tamargo P, Estenoz J. Estudio de morbilidad y mortalidad en el tromboembolismo pulmonar. Rev Cubana Angiol Cir Vasc. 2006; 7(1). 
12-Tami de Araujo MD, Valencia I, Federman D, Kirsner R. Managing the patient with venous ulcers. Ann Intern Med. 2003; 138: 326-34.

13- Monreal M, Barba R, Tolosa C, Tiberio G, Todoli J, Samperiz AL, et al. Deep vein thrombosis and pulmonary embolism: the same disease? Pathophysiol Haemost Thromb. 2006; 35(1-2): 133-5.

Recibido: 24 de septiembre de 2007.

Aprobado: 3 de octubre de 2007.

Ulises de Jesús Gallardo Pérez. Instituto Nacional de Angiología y Cirugía Vascular. Telef.: 870-46-30. Email: epidema@infomed.sld.cu /HTML>

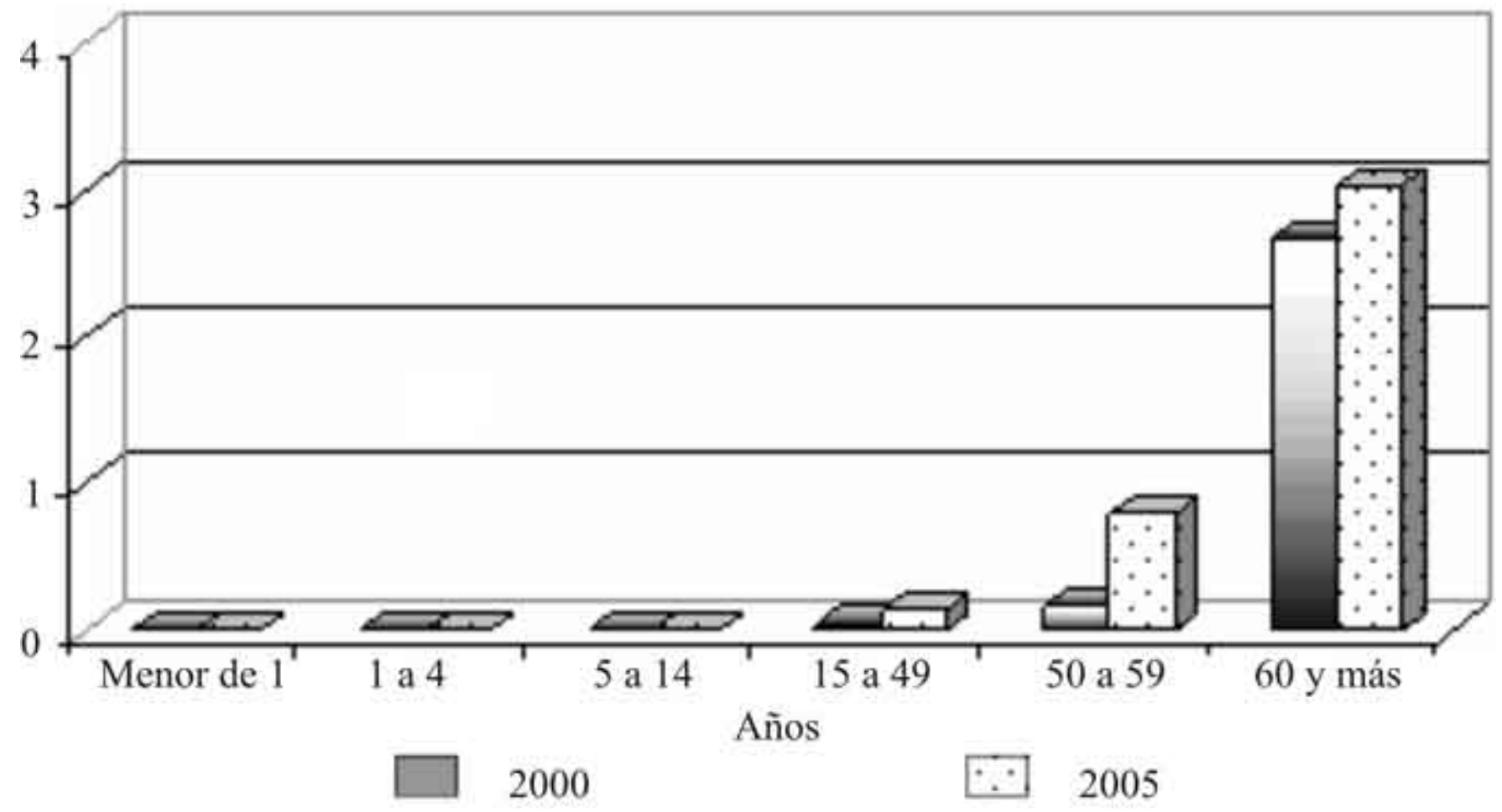

Fig. 1. Mortalidad por flebitis y tromboflebitis según grupos de edades, Cuba 2000 y 2005. 


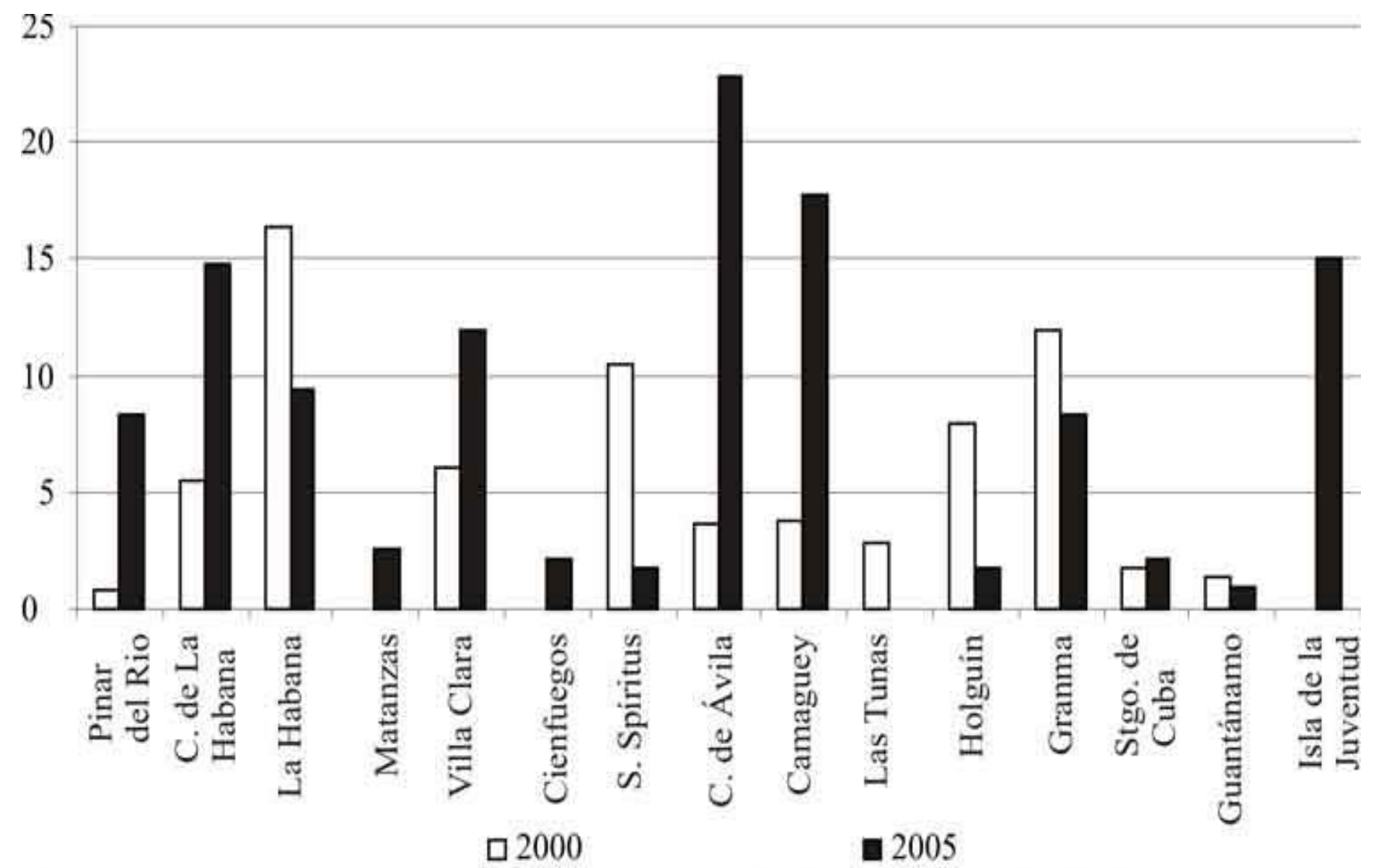

Fig. 2. Carga por muertes prematuras, flebitis y tromboflebitis según provincias, Cuba 2000 y 2005. 